\title{
Hippocampal and Amygdalar Volumetric Differences in Pathological Gambling: a Preliminary Study of the Associations with the Behavioral Inhibition System
}

\author{
Ardeshir S Rahman', Jiansong $X \mathbf{u}^{\prime}$ and Marc N Potenza*, I,2,3 \\ 'Connecticut Mental Health Center, Department of Psychiatry, Yale University School of Medicine, New Haven, CT, USA; ${ }^{2}$ Child Study \\ Center, Yale University, School of Medicine, New Haven, CT, USA; ${ }^{3}$ Department of Neurobiology, Yale University, School of Medicine, \\ New Haven, CT, USA
}

\begin{abstract}
The behavioral inhibition system (BIS) and behavioral activation system (BAS) are hypothesized to underlie motivated behavior, relate to hippocampal and amygdalar function, and link to pathological gambling (PG). Prior studies have not investigated hippocampal and amygdalar volumes in PG and their relationships to BIS/BAS measures. Structural MRI scans and BIS/BAS and other clinical measures were obtained from 32 PG individuals and 47 healthy comparison $(\mathrm{HC})$ individuals. Volumetric measures of the hippocampus and amygdala were assessed using FreeSurfer and related to BIS/BAS measures. PG relative to HC individuals demonstrated diminished volume in the left hippocampus and right amygdala and higher BIS and BAS scores. BIS scores were positively correlated with left hippocampal and left amygdalar volumes in PG individuals. The findings of relatively diminished hippocampal and amygdalar volumes in PG individuals resonate with findings from substance-dependent groups. Relationships between amygdalar and hippocampal volumes and BIS measures in PG suggest that individual differences in these structures may contribute to avoidance behaviors in PG.

Neuropsychopharmacology (2014) 39, 738-745; doi:I0.1038/npp.2013.260; published online 23 October 2013
\end{abstract}

Keywords: pathological gambling; behavioral inhibition; behavioral activation; brain volume; hippocampus; amygdala

\section{INTRODUCTION}

Gray's theory regarding the neural pathways that underlie motivated behaviors encompasses two parallel systems-a behavioral inhibition system (BIS) and a behavioral activation system (BAS; Gray, 1987). According to Gray, the BIS is sensitive to punishment, novelty, and negative affective states including anxiety and depression (Gray, 1987; Gray and McNaughton, 2000). It has been postulated that the neural correlates of the BIS include the amygdala and septo-hippocampal systems, with high activity in these areas associated with anxiety (Gray, 1987; Gray and McNaughton, 2000). Conversely, the BAS is sensitive to reward signals and is associated with positive affective states like happiness and elation (Carver and White, 1994; Gray, 1987). Gray proposed the BAS to be imbedded primarily in the septal area and the hypothalamus (Gray, 1987). However, the neural underpinnings of the BAS are not as well elaborated as the BIS, although dopaminergic pathways may contribute (Carver and White, 1994; Depue and Collins, 1999; Gray, 1987).

* Correspondence: Professor MN Potenza, Connecticut Mental Health Center, Department of Psychiatry and Child Study Center, Yale University School of Medicine, I Church St, 7th Floor Room 726, New Haven, CT 065 19, USA, Tel: + | 203737 3553, Fax: + | 203737 359|, E-mail: marc.potenza@yale.edu

Received II April 2013; revised 6 September 2013; accepted 8 September 2013; accepted article preview online 27 September 2013
Structural studies investigating reward and risk assessment and emotionally motivated behavior support the BIS/BAS function in regions initially proposed by Gray. For example, voxel-based-morphometry studies found that hippocampal and amygdalar volumes were positively correlated with BIS activity, whereas dorsal striatal and superior frontal gyral volumes were negatively correlated with BAS activity (Barros-Loscertales et al, 2006a, b). Additionally, larger hippocampal volumes have been associated with increased anxiety, whereas reduced hippocampal volumes have been implicated in increased impulsivity (Rusch et al, 2001; Zetzsche et al, 2007). In a large sample $(n=430)$ of healthy adults, BIS activity correlated with hippocampal volumes (Cherbuin et al, 2008). These studies support a relationship between hippocampal and amygdalar volume and BIS/BAS function.

BIS/BAS function is relevant to multiple psychiatric disorders. For example, increased BIS activation and decreased BAS activation have been associated with depression and anxiety (Hundt et al, 2007; Johnson et al, 2003). BAS-reward-responsiveness (BAS-RR) and BASdrive (BAS-D) subscale scores are low in depressed adults (McFarland et al, 2006). Substance-use disorders have also been associated with BIS/BAS function (although see van Toor et al (2011)), with BAS-fun-seeking (BAS-FS) and BAS-D activity associated with the substance use and/or lifetime drug dependence (Franken et al, 2006; Johnson et al, 2003), and BIS function inversely related to binge-drinking 
behavior. BIS, BAS-RR, and BAS-FS activity have also been positively associated with impulsivity and hazardous drinking in a community sample of adults (Hamilton et al, 2012).

Pathological gambling (PG) may also be considered within this framework. PG is characterized by impaired impulse control and altered risk assessment, which suggests that BIS/BAS may relate to PG (Potenza, 2008; Potenza et al, 2003; Tanabe et al, 2007). PG is associated with poor physical and psychiatric health in both adults and adolescents (Potenza et al, 2001; Rahman et al, 2012; Shaffer and Korn, 2002). As gambling in PG may be motivated by negative or positive reinforcement tendencies, BIS and BAS systems may relate importantly to PG as they do to substance-use disorders (Grant and Kim, 2002).

Several studies have described the relationship between BIS/BAS function and gambling behaviors. Among college students, negative affect was associated with problemgambling severity and with scores on the BIS assessment, whereas BAS-RR was inversely correlated with problemgambling severity (Atkinson et al, 2012). Problem gamblers also score higher on the BIS and BAS scales, with higher BIS/ BAS scores associated with worse performance on the Iowa Gambling Task (Goudriaan et al, 2006). In non-problematic adult gamblers, BIS scores were more closely related to risky betting than were BAS scores (Demaree et al, 2008). PG individuals also scored higher than healthy comparison (HC) subjects on the BAS-FS and BAS-D scales, with BAS-FS scores inversely correlated with the white matter integrity in the left and right genu of the corpus callosum (Yip et al, 2013).

Although hippocampal and amygdalar volumes have been linked to BIS and PG to BIS/BAS measures, no studies have investigated brain volumes in $\mathrm{PG}$ as related to BIS/BAS measures. Few studies have investigated brain volumetric differences in PG and HC subjects, with one small study finding no between-group differences (Joutsa et al, 2011; Leeman and Potenza, 2012). However, studies of other groups with addictions (eg, those with cocaine dependence) have shown smaller hippocampal and amygdalar volumes in addicted vs comparison samples (Makris et al, 2004; Rando et al, 2013). Here we sought to examine volumetric measures in a larger sample of PG and $\mathrm{HC}$ subjects and explore relationships with BIS/BAS. We hypothesized that: (1) PG vs HC participants would score higher on BIS and BAS scales; (2) PG vs HC participants would have diminished hippocampal and amygdalar volumes; and (3) Hippocampal and amygdalar volumes would positively associate with BIS activity, particularly within PG participants.

\section{MATERIALS AND METHODS}

\section{Participants}

Both HC and non-treatment-seeking PG individuals were recruited via advertising. Final enrollment included 32 PG (12 female) and 47 HC (19 female) individuals. Participants were assessed using the Structured Clinical Interview for DSM-IV (SCID; First et al, 1995). PG status was determined using the Structured Clinical Interview for Pathological Gambling (SCI-PG; Grant et al, 2004). Exclusion criteria for this study included: current or planned pregnancy, current non-substance-use Axis I disorders, or unstable medical conditions. All participants provided written informed consent before enrolling and all study procedures were approved by the Yale Human Investigation Committee.

Demographic data collected included: age, gender, race/ ethnicity, and education. Smoking status was categorized as either current smoker or not. Alcohol and drug dependence were assessed during structured interviews. Drug categories included sedatives, marijuana, stimulants, opiates, cocaine, hallucinogens, and 'other.' The HC group had no individuals that had current or prior alcohol or drug dependence, whereas the PG group had 11 individuals, including one each with current alcohol and cocaine dependence. The specific prior disorders in the PG group included one individual with prior alcohol dependence, cocaine dependence, and sedative/hypnotic dependence (in remission from all for greater than a year), two individuals with prior opiate dependence (in remission for over 2 years and 10 years, respectively), one individual with prior alcohol dependence (in remission for 9 years), one individual with prior cocaine dependence (in remission for 19 years), one individual with prior alcohol dependence, cannabis dependence and cocaine dependence (in remission for 7 months), one individual with prior cannabis dependence (in remission for 4 months), one individual with prior alcohol and cocaine dependence (in remission for 7 years), and one individual with prior alcohol, opiate and cocaine dependence (in remission for 10 years, 7 years and 4 months, respectively).

\section{Measures}

The BIS and BAS (BIS/BAS) Scale. The BIS/BAS scale is a reliable and valid 24-item scale that measures behavioral inhibition and approach (Carver and White, 1994; Jorm et al, 1998). The scale assesses behavioral tendencies with regard to risk aversion or reward seeking. Participants select from a Likert-style scale the degree to which they agree or disagree with the statements. The measure generates two subscale scores relating to BIS and BAS. The BIS measures inhibition to negative affective outcomes, whereas the BAS measures response to positive affective outcomes (Carver and White, 1994). High BIS and BAS scores reflect high BIS and BAS activity, respectively.

The alcohol use disorders identification test. The alcohol use disorders identification test (AUDIT) is a valid and reliable 10-item screen that identifies hazardous alcohol use (Babor et al, 2001). Respondents can select between five response choices which are given specific scores, with higher total scores associated with increased severity.

The beck anxiety inventory. The Beck Anxiety Inventory (BAI) is a valid and reliable 21-item instrument that assesses anxiety (Beck et al, 1993). Respondents select between four responses per question, and higher total scores reflect increased severity.

The beck depression inventory II. The beck depression inventory II (BDI) is a valid and reliable 21-item instrument that assesses depression (Beck et al, 1996). Respondents select between four responses per question, and higher total scores reflect increased severity. 


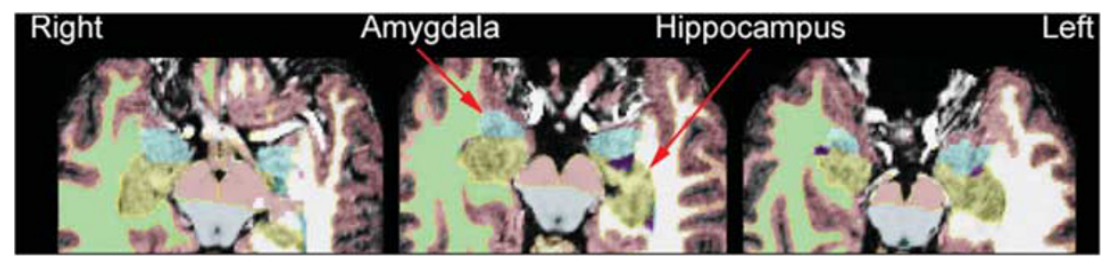

Figure I Volumetric differences in mesial temporal structures. Both right amygdalar and left hippocampal volumes were significantly decreased in pathological gambling (PG) subjects $(n=32)$ compared with healthy comparison (HC) subjects $(n=47)$. High-resolution TI-weighted anatomical images were acquired using a 3-T scanner (Trio; Siemens) and mesial temporal structures outlined using FreeSurfer software (http://wmw.surfer.nmr.mgh.harvard.edu).

\section{Image Acquisition and Processing}

High-resolution T1-weighted anatomical images were acquired using a 3-T scanner (Trio; Siemens, Erlangen, Germany) with the following parameters: $\mathrm{TR}=1500 \mathrm{~ms}, \mathrm{TE}=2.83 \mathrm{~ms}$, flip angle $=7^{\circ}, \mathrm{FOV}=256 \times 256 \mathrm{~mm}^{2}$, matrix $=256 \times 256,1 \mathrm{~mm}^{3}$ isotropic voxels, 176 slices. The hippocampus and amygdala were automatically segmented using the software FreeSurfer version 5.1.0 (http://surfer.nmr.mgh.harvard.edu) (Fischl et al, 2001) (Figure 1). The validity and reliability of the FreeSurfer package have been found to be very good (Morey et al, 2009; Shen et al, 2010). FreeSurfer scripts autorecon1, 2, and 3 were run in sequence on all imaging data. In brief, processing consisted of removal of the non-brain tissue, Talairach transformation, segmentation of subcortical volumetric structures including hippocampus and amygdala, intensity normalization, tessellation of the gray-matter/white-matter boundary, and labeling of each voxel based on previous probabilistic information. Intracranial volume was generated during this processing. The detailed procedure has been described previously (Fischl et al, 2002).

\section{Data Analysis}

All data were double-entered and randomly spot-checked for accuracy. Subsequent analyses were conducted in SPSS (version 20.0; IBM; Chicago, IL). Gender comparisons between groups utilized $\chi^{2}$ analysis and showed the groups to be well matched. Means for age and education were analyzed using $t$-tests. Mean volumes were compared using the general linear model, correcting for age, education, and intracranial volume. Gender was typically not included as a covariate given that groups were evenly matched. All scores on behavioral assessments (BDI, BAI, BAS, and BIS) were compared using the general linear model and adjusted for age and years of education. Partial correlations were conducted between volumes and relevant behavioral assessments, correcting for age, and years of education. Multiple regression models were created for BIS and BAS scores and split by clinical group (HC and PG). All models included age, gender, years of education, intracranial volume, BAI scores, BDI scores, left amygdalar volume, right amygdalar volume, left hippocampal volume, and right hippocampal volume as predictor variables. Additionally, for the PG group, AUDIT score, past/present smoking status, past/present alcohol dependence, and past/present drug dependence were also included as predictor variables. Statistical significance was set at $P<0.05$ unless otherwise indicated.

\section{RESULTS}

\section{Demographics}

Most PG and comparison participants were male: $62.5 \%$ and $59.6 \%$, respectively (Table 1 ). There was no between-group difference in gender $\left(x^{2}=0.068, P=0.794\right)$. PG individuals differed from healthy individuals on age $(t=-2.78$, $P=0.007)$ and education $(t=4.40, P<0.001)$, with $\mathrm{PG}$ individuals being older and having less education.

\section{Clinical Measures}

PG individuals scored higher on the AUDIT $(F=12.00$, $P=0.001), \quad$ BDI $\quad(\mathrm{F}=18.75, P<0.001), \quad$ BAS $\quad(\mathrm{F}=11.618$, $P=0.001)$, and BIS $(\mathrm{F}=4.47, P=0.039)$ (Table 1$)$.

\section{Hippocampal and Amygdalar Volumes}

PG individuals had smaller volumes in the right amygdala $(\mathrm{F}=4.31, \quad P=0.041)$ and left hippocampus $(\mathrm{F}=5.49$, $P=0.022$ ) (Table 1). PG individuals did not differ from healthy individuals in intracranial volume $(\mathrm{F}=0.984$, $P=0.325)$, left amygdalar volume $(F=2.91, P=0.092)$, or right hippocampal volume $(\mathrm{F}=2.33, P=0.131)$.

\section{Correlations Between Brain Volumes and Clinical Assessments}

No significant correlations were observed in HC subjects (Supplementary Table 1). For PG individuals, a positive correlation was detected between BIS scores and left amygdalar volumes $(r=0.606, P<0.01)$ and BIS scores and left hippocampal volumes $(r=0.457, P<0.05)$.

\section{Multiple Regression Analyses}

For the HC group, volumes of the amygdala and hippocampus were not related to BIS nor BAS scale scores when adjusting for the modeled variables including age, gender, years of education, intracranial volume, and BAI and BDI scores (Table 2). For the PG group, left amygdalar volumes were related to BIS scores $(\beta=0.846, P=0.038)$, and the relationship between right hippocampal volumes and BIS scores strengthened but did not reach significance at $p<0.05 \quad(\beta=0.728, \quad P=0.082) \quad$ when adjusting for variables including age, gender, years of education, intracranial volume, BAI, BDI, AUDIT, past/present smoking status, past/present alcohol dependence, and past/ present drug dependence (Table 3). The relationship between BIS scores and left hippocampal volumes in 
unadjusted analyses $(r=0.475, P<0.05)$ was no longer observed in the adjusted model $(\beta=-0.132, P=0.703)$.

\section{DISCUSSION}

This study investigated hippocampal and amygdalar volumes and BIS/BAS tendencies in PG and HC subjects. Partially consistent with our a priori hypotheses, PG subjects showed relatively smaller hippocampal and amygdalar volumes, with findings reaching statistical significance in the right amygdala and left hippocampus. As hypothesized, PG subjects scored higher than HC subjects on the BIS/BAS scales, and partially consistent with our hypotheses, left amygdalar, and hippocampal volumes correlated with BIS scores in PG but not in HC subjects. When covarying for clinical factors, findings persisted between left amygdalar volumes and BIS scores in PG subjects. Clinical implications are discussed below.

Table I Demographic, Volumetric, and Behavioral Differences between Groups

\begin{tabular}{|c|c|c|c|c|c|}
\hline \multirow[t]{2}{*}{ Demographics } & $\mathrm{HC}(n=47)$ & PG $(n=32)$ & \multirow[t]{2}{*}{ df } & \multirow[t]{2}{*}{$x^{2}$} & \multirow[t]{2}{*}{$P$} \\
\hline & \multicolumn{2}{|c|}{$N(\%)$} & & & \\
\hline \multirow[t]{2}{*}{ Females } & $19(40.4 \%)$ & $12(37.5 \%)$ & I & 0.068 & 0.794 \\
\hline & \multicolumn{2}{|c|}{ Mean values } & $d f$ & $t$ & $P$ \\
\hline Age (years) & 29.62 & 36.47 & 77 & -2.787 & 0.007 \\
\hline Education (years) & 14.72 & 13.03 & 77 & 4.404 & $<0.001$ \\
\hline Volumes & \multicolumn{2}{|c|}{ Mean values } & $\mathrm{df}$ & $F$ & $P$ \\
\hline $\mathrm{ICV}(I)^{\mathrm{a}}$ & 1.073 & 1.066 & I & 0.984 & 0.325 \\
\hline LA $(m l)^{b}$ & 1.729 & 1.602 & 1 & 2.912 & 0.092 \\
\hline $\mathrm{RA}(\mathrm{ml})^{\mathrm{b}}$ & 1.828 & 1.715 & 1 & 4.318 & 0.041 \\
\hline $\mathrm{LH}(\mathrm{ml})^{\mathrm{b}}$ & 4.061 & 3.873 & 1 & 5.495 & 0.022 \\
\hline $\mathrm{RH}(\mathrm{ml})^{\mathrm{b}}$ & 4.201 & 4.034 & 1 & 2.337 & 0.131 \\
\hline \multicolumn{6}{|l|}{ Behavioral } \\
\hline AUDIT ${ }^{\mathrm{a}}$ & 3.240 & 6.140 & 1 & 12.003 & 0.001 \\
\hline $\mathrm{BAl}^{\mathrm{a}}$ & 3.850 & 5.760 & 1 & 2.930 & 0.092 \\
\hline $\mathrm{BDI}^{\mathrm{a}}$ & 2.37 & 9.14 & 1 & 18.752 & $<0.001$ \\
\hline BAS total ${ }^{\mathrm{a}}$ & 37.68 & 42.38 & 1 & 11.618 & 0.001 \\
\hline BIS total ${ }^{\mathrm{a}}$ & 18.02 & 19.86 & 1 & 4.471 & 0.039 \\
\hline
\end{tabular}

Abbreviations: AUDIT, Alcohol Use Disorders Identification Test; BAl, Beck Anxiety Inventory; BDI, Beck Depression Inventory; BAS, behavioral activation system; BIS, behavioral inhibition system; ICV, intracranial volume; $\mathrm{HC}$, healthy controls; PG, problem/pathological gamblers; LA, left amygdala; RA, right amygdala; $\mathrm{LH}$, left hippocampus; $\mathrm{RH}$, right hippocampus.

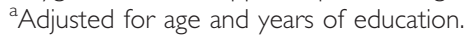

${ }^{\mathrm{b}}$ Adjusted for age, years of education, and intracranial volume.

\section{Behavioral Tendencies-BIS/BAS Scores}

Consistent with our first hypothesis and with prior findings in problem gamblers, PG individuals scored higher on BIS and BAS measures (Goudriaan et al, 2006). Higher BIS scores in the PG group indicate greater BIS activity (Carver and White, 1994; Gray, 1987). As the BIS works to inhibit behaviors that may result in negative outcomes, greater activity is associated with tendencies to inhibit behaviors and might reflect internalizing features observed in PG, as is evidenced by frequent co-occurrence of depression and anxiety disorders (Giddens et al, 2012; Potenza et al, 2005). Moreover, PG individuals score higher on the BAS, suggesting higher activity in this system. Within Gray's proposed motivational framework, the BIS and BAS are considered separate systems, however, they are not diametrically opposed and are, perhaps, more orthogonal as evinced by the current findings and others finding BIS and BAS scores positively relating to the AUDIT scores (Hamilton et al, 2012). Accordingly, higher BIS and BAS scores indicate that $\mathrm{PG}$ individuals are not only more inclined to demonstrate greater avoidance but also more highly motivated by external rewards (Johnson et al, 2003). Consistent with this notion, during gambling tasks (Cavedini et al, 2002; Goudriaan et al, 2006; Petry, 2001; van Holst et al, 2010), PG individuals typically make risky decisions for monetary rewards despite potential negative outcomes. The replication in PG of findings previously observed in problem gamblers suggests BIS/BAS tendencies relate to excessive gambling across diagnostic boundaries, cultures, and geographic locations and imply that both avoidance and approach tendencies relate to PG, perhaps through motivational tendencies underlying gambling behaviors.

\section{Hippocampal and Amygdalar Volumes}

In support of our second hypothesis, PG individuals showed relatively decreased hippocampal and amygdalar volumes. These findings are consistent with those in other addictive disorders like stimulant dependence (Makris et al, 2004; Rando et al, 2013). Additionally, the findings are consistent with an association between PG and elevated stress exposure (Elman et al, 2010) and stress and smaller hippocampal volumes (Gilbertson et al, 2002). To our knowledge, only one study has investigated volumetric differences in PG and showed no between-group differences, perhaps as the study involved a small sample (Joutsa et al, 2011).

The positive correlation between BIS scores and hippocampal volumes reflects a general relationship observed in non-PG populations (Cherbuin et al, 2008). In a model adjusting for clinically relevant variables (including those

Table 2 Multiple Regression Analyses Describing the Effects of Relevant Variables on BIS/BAS Scores-HC

\begin{tabular}{|c|c|c|c|c|c|c|c|c|c|c|}
\hline & Age & Gender & Edu & ICV & BAI & BDI & LA & RA & LH & $\mathbf{R H}$ \\
\hline BAS total & $-0.303(0.061)$ & $0.14(0.542)$ & $0.223(0.18)$ & $-0.574(0.037)$ & $-0.105(0.522)$ & $-0.067(0.682)$ & $-0.02(0.927)$ & $0.395(0.122)$ & $0.331(0.234)$ & $-0.268(0.295)$ \\
\hline
\end{tabular}

Abbreviations: BAI, Beck Anxiety Inventory; BDI, Beck Depression Inventory; Edu, education; ICV, intracranial volume; HC, healthy comparison; LA, left amygdala; LH, left hippocampus; RA, right amygdala; RH, right hippocampus. 
relating to substance-use disorders), the association between BIS scores and left hippocampal volumes weakened and that between BIS scores and right hippocampal volumes strengthened, suggesting that co-occurring substance-use exposure/behaviors/disorders may influence/and/or alter laterality relationships between hippocampal volumes and BIS tendencies, although this possibility warrants further examination. The positive direction of the correlations suggests that greater hippocampal volume is associated with greater behavioral inhibition, as assessed through the BIS scale. Thus, although the PG group as a whole exhibited greater BIS scores and smaller hippocampal volumes, the tendency on an individual basis for larger volumes to associate with greater BIS activity was observed in the PG sample as has been reported in non-PG groups. This raises the possibility that the overall group effect relating to elevated BIS scores in PG may not be driven by hippocampal volumes (with perhaps other mechanisms underlying a group effect), but rather that individual differences in BISrelated tendencies in PG subjects relate positively to hippocampal volume as has been observed in other populations. The most robust correlation within the PG group was between BIS scores and left amygdalar volume and is also consistent with prior studies in which PG was not assessed (Barros-Loscertales et al, 2006a), suggesting that this pattern relating behavioral activation to amygdalar volumes reflects a more general relationship across groups, whereas the between-group difference relating to PG diagnostic status displays a different relationship. Identification of structural or functional influences that might be driving the group effects in BIS measures remains an important endeavor. Clinically, the finding suggests that individual differences relating to BIS tendencies within PG may be similar to those in the general population and might respond to interventions targeting the BIS more generally.

\section{Limitations and Future Directions}

Several limitations exist. First, although the sample is considerably larger than in a prior PG report (Joutsa et al, 2011), the sample size remains relatively modest, which may impact power and generalizability. It may be that some variables that approached significance might become significant if a larger sample was studied. Additionally, although all statistical analyses corrected for age, the HC group was younger than the PG group, and age has been related to amygdalar and hippocampal volumes. Future investigations should investigate age-matched samples of PG and HC subjects, and ultimately study groups of varying ages. Moreover, given the preliminary nature of this study (particularly with respect to BIS/BAS relationships), a liberal threshold was used in analyses, which could possibly lead to false discovery. None of the current findings survive a Bonferroni correction, with the most robust finding (between left amygdalar volume and BIS scores) significant at $p<0.01$. Future studies employing larger and bettermatched samples are indicated to seek to replicate and extend the current findings. Although this study's hypotheses focused on hippocampal and amygdalar volumes, future studies should also consider examining whole-brain differences between PG and HC subjects. Furthermore, selfreports were used to assess behavioral tendencies. Although 
a
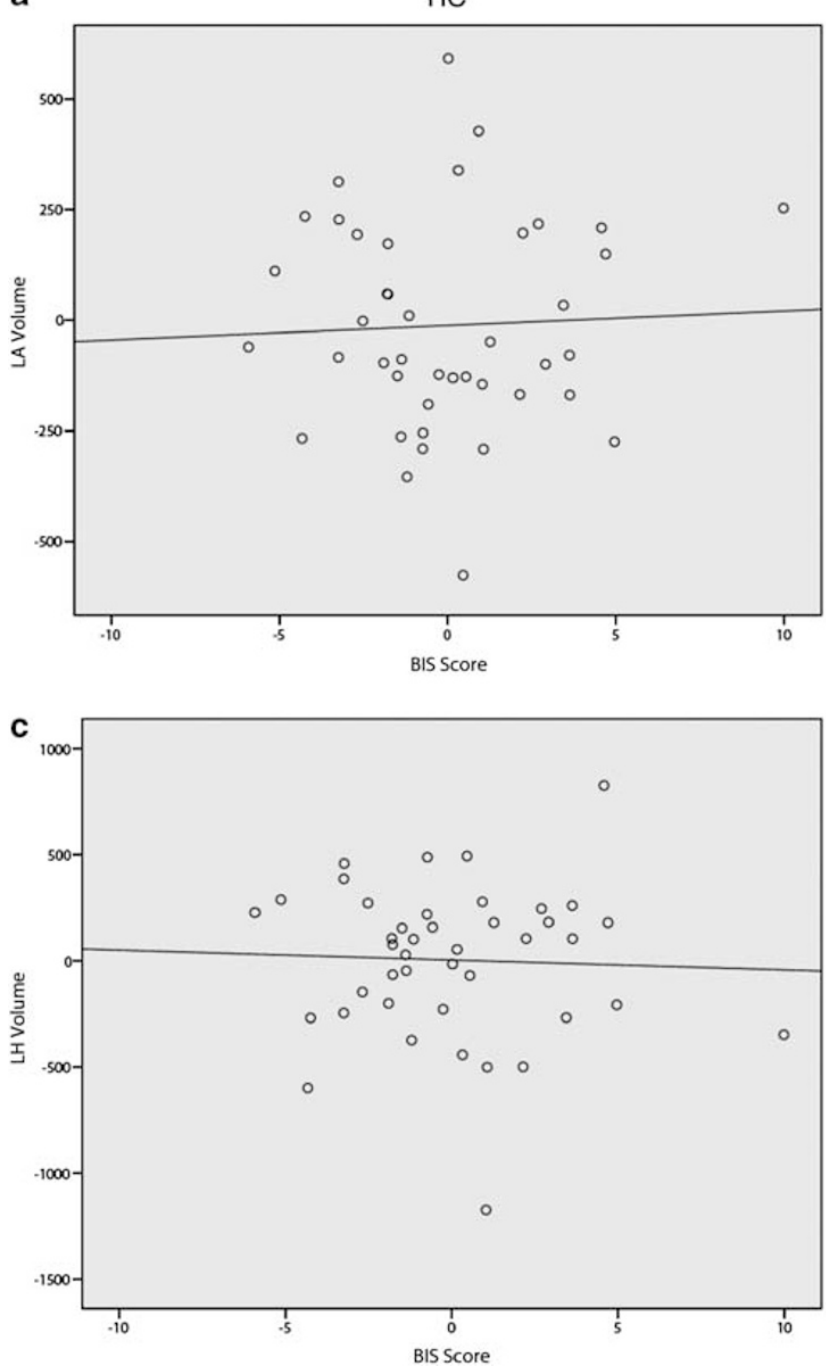

b
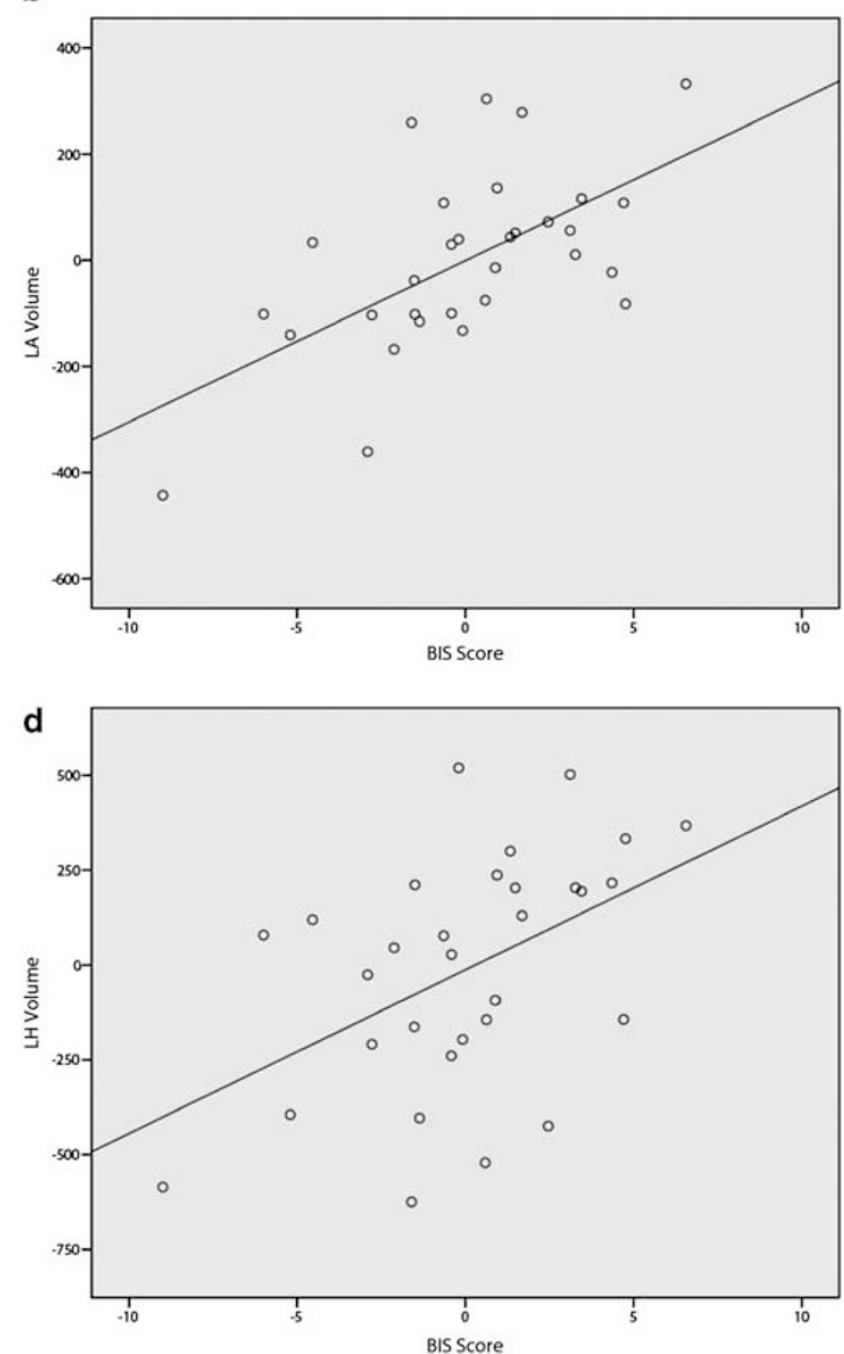

Figure 2 Correlations between behavioral assessments and structural volumes in both HC and pathological gambling (PG) subjects. Although there was no correlation between behavioral inhibition system (BIS) and left amygdalar volume in healthy comparison (HC) subjects (a), a positive correlation was observed in PG subjects (b). Similarly, there was no correlation between BIS and left hippocampal volume in HC subjects (c), but there was a positive correlation in PG subjects (d). Plots were generated using residual values after controlling for age, years of education, and intracranial volume.

the scales employed have been shown to be valid, reliable, and yield valuable clinically relevant information (Babor et al, 2001; Beck et al, 1996; Beck et al, 1993; Carver and White, 1994), future studies should examine hippocampal and amygdalar function with respect to actual behaviors in individuals with PG. Additionally, future studies should investigate the clinical implications of the observed findings. For example, the relationship between amygdalar and hippocampal volumes as related to treatment outcome should be examined. Prior studies have found that among individuals with depression, those with smaller hippocampal volumes demonstrated poorer treatment outcome prospectively (Frodl et al, 2008). Although brain activation measures relating to cognitive control have been preliminary linked to treatment outcome in PG (Potenza et al, 2013), future imaging studies employing larger samples are needed to investigate the biological factors underlying vulnerability and biological mechanisms underlying clinical improvement. Finally, the factors leading to smaller hippocampal and amygdalar volumes should be studied. For example, if stress exposure might underlie the finding of smaller hippocampal volumes in PG, then early interventions that reduce stress might provide protection from neural insults in people with $\mathrm{PG}$.

\section{CONCLUSIONS}

The current study is the first to our knowledge to identify between-group differences in brain volumes in PG as compared with HC subjects. Additional research is needed to determine the extent to which smaller hippocampal and amygdalar volumes might result from or confer vulnerability to PG, and the extent to which differences in hippocampal and amygdalar volumes relate to clinically relevant features of PG-like treatment outcomes.

\section{FUNDING AND DISCLOSURE}

This research was funded in part by NIH grants from NIDA (K01 DA027750, P20 DA027844, R01 DA019039), NIAAA 
(RL1 AA017539), the Connecticut State Department of Mental Health and Addictions Services, the Connecticut Mental Health Center, an unrestricted research gift from the Mohegan Sun casino, and the Yale Gambling Center of Research Excellence Award grant from the National Center for Responsible Gaming. The funding agencies did not provide input or comment on the content of the manuscript, and the content of the manuscript reflects the contributions and thoughts of the authors and do not necessarily reflect the views of the funding agencies. Dr Potenza has consulted for Lundbeck and Ironwood pharmaceuticals; has had financial interests in Somaxon pharmaceuticals; received research support from Mohegan Sun Casino, Psyadon pharmaceuticals, the National Center for Responsible Gambling, the National Institutes of Health (NIH), Veterans Administration; has participated in surveys, mailings, or telephone consultations related to drug addiction, impulse-control disorders, or other health topics; has consulted for gambling, legal and governmental entities on issues related to addictions or impulse-control disorders; has provided clinical care in the Connecticut Department of Mental Health and Addiction Services Problem Gambling Services Program; has performed grant reviews for the NIH and other agencies; has guest edited journal sections; has given academic lectures in grand rounds, Continuing Medical Education events, and other clinical or scientific venues; and has generated books or book chapters for publishers of mental health texts. Dr Xu and Mr Rahman have no disclosures.

\section{ACKNOWLEDGEMENTS}

We thank Scott Bullock, Monica Solorzano, Stephen Healy, Jessica Montoya, Katherine VanBuskirk, Michale Bernabeo, Jennifer Bellegarde, and Jocelyn Topf for their involvement in data collection.

\section{REFERENCES}

Atkinson J, Sharp C, Schmitz J, Yaroslavsky I (2012). Behavioral activation and inhibition, negative affect, and gambling severity in a sample of young adult college students. J Gambl Stud 28: 437-449.

Babor T, Higgins-Biddle JC, Saunders J, Monteiro M (2001). AUDIT-The Alcohol Use Disorders Identification Test: Guidlines for Use in Primary Care. 2nd edn. Department of Mental Health and Substance Dependence, World Health Organization: Geneva, Switzerland.

Barros-Loscertales A, Meseguer V, Sanjuan A, Belloch V, Parcet MA, Torrubia R et al (2006a). Behavioral inhibition system activity is associated with increased amygdala and hippocampal gray matter volume: A voxel-based morphometry study. NeuroImage 33: 1011-1015.

Barros-Loscertales A, Meseguer V, Sanjuan A, Belloch V, Parcet MA, Torrubia R et al (2006b). Striatum gray matter reduction in males with an overactive behavioral activation system. Eur J Neurosci 24: 2071-2074.

Beck AT, Steer RA, Ball R, Ranieri W (1996). Comparison of beck depression inventories -IA and -II in psychiatric outpatients. J Pers Assess 67: 588-597.

Beck AT, Steer RA, Beck JS (1993). Types of self-reported anxiety in outpatients with DSM-IIIR anxiety disorders. Anxiety Stress Coping 6: 43-55.
Carver CS, White TL (1994). Behavioral-Inhibition, behavioral activation, and affective responses to impending reward and punishment-the Bis Bas Scales. J Pers Soc Psychol 67: 319-333.

Cavedini P, Riboldi G, Keller R, D’Annucci A, Bellodi L (2002). Frontal lobe dysfunction in pathological gambling patients. Biol Psychiatry 51: 334-341.

Cherbuin N, Windsor TD, Anstey KJ, Maller JJ, Meslin C, Sachdev PS (2008). Hippocampal volume is positively associated with behavioural inhibition (BIS) in a large community-based sample of mid-life adults: the PATH through life study. Soc Cogn Affect Neurosci 3: 262-269.

Demaree HA, DeDonno MA, Burns KJ, Erik Everhart D (2008). You bet: how personality differences affect risk-taking preferences. Pers Individ Dif 44: 1484-1494.

Depue RA, Collins PF (1999). Neurobiology of the structure of personality: dopamine, facilitation of incentive motivation, and extraversion. Behav Brain Sci 22: 491-517 discussion 518-469.

Elman I, Tschibelu E, Borsook D (2010). Psychosocial stress and its relationship to gambling urges in individuals with pathological gambling. Am J Addict 19: 332-339.

First M, Spitzer R, Gibbon M, Williams J (1995). Structured Clinical Interview for DSM-IV. Patient edn American Psychiatric Press Inc.: Washington, DC, USA.

Fischl B, Liu A, Dale AM (2001). Automated manifold surgery: constructing geometrically accurate and topologically correct models of the human cerebral cortex. IEEE Trans Med Imaging 20: 70-80.

Fischl B, Salat DH, Busa E, Albert M, Dieterich M, Haselgrove C et al (2002). Whole brain segmentation: automated labeling of neuroanatomical structures in the human brain. Neuron 33: 341-355.

Franken IH, Muris P, Georgieva I (2006). Gray's model of personality and addiction. Addict Behav 31: 399-403.

Frodl T, Jager M, Smajstrlova I, Born C, Bottlender R, Palladino T et al (2008). Effect of hippocampal and amygdala volumes on clinical outcomes in major depression: a 3-year prospective magnetic resonance imaging study. J Psychiatry Neurosci 33: 423-430.

Giddens JL, Stefanovics E, Pilver CE, Desai R, Potenza MN (2012). Pathological gambling severity and co-occurring psychiatric disorders in individuals with and without anxiety disorders in a nationally representative sample. Psychiatry Res 199: 58-64.

Gilbertson MW, Shenton ME, Ciszewski A, Kasai K, Lasko NB, Orr SP et al (2002). Smaller hippocampal volume predicts pathologic vulnerability to psychological trauma. Nat Neurosci 5: 1242-1247.

Goudriaan AE, Oosterlaan J, de Beurs E, van den Brink W (2006). Psychophysiological determinants and concomitants of deficient decision making in pathological gamblers. Drug Alcohol Depend 84: 231-239.

Grant JE, Kim SW (2002). Gender differences in pathological gamblers seeking medication treatment. Compr Psychiatry 43: 56-62.

Grant JE, Steinberg MA, Kim SW, Rounsaville BJ, Potenza MN (2004). Preliminary validity and reliability testing of a structured clinical interview for pathological gambling. Psychiatry Res 128: 79-88.

Gray JA (1987). Cognitive Neurochemistry. Oxford University Press: New York, NY, USA.

Gray JA, McNaughton N (2000). The Neuropsychology of Anxiety. 2 edn. Oxford University Press: New York, NY, USA.

Hamilton KR, Sinha R, Potenza MN (2012). Hazardous drinking and dimensions of impulsivity, behavioral approach, and inhibition in adult men and women. Alcohol Clin Exp Res 36: 434-449.

Hundt NE, Nelson-Gray RO, Kimbrel NA, Mitchell JT, Kwapil TR (2007). The interaction of reinforcement sensitivity and life events in the prediction of anhedonic depression and mixed anxiety-depression symptoms. Pers Individ Dif 43: 1001-1012.

Johnson SL, Turner RJ, Iwata N (2003). BIS/BAS levels and psychiatric disorder: an epidemiological study. J Psychopathol Behav Assessment 25: 25-36. 
Jorm AF, Christensen A, Henderson P, Jacomb A (1998). Using the BIS/BAS scales to measure behavioural inhibition and behavioural activation: factor structure, validitiy and norms in a large community sample. Pers Individ Dif 26: 49-58.

Joutsa J, Saunavaara J, Parkkola R, Niemela S, Kaasinen V (2011). Extensive abnormality of brain white matter integrity in pathological gambling. Psychiatry Res 194: 340-346.

Leeman RF, Potenza MN (2012). Similarities and differences between pathological gambling and substance use disorders: a focus on impulsivity and compulsivity. Psychopharmacology (Berl) 219: 469-490.

Makris N, Gasic GP, Seidman LJ, Goldstein JM, Gastfriend DR, Elman I et al (2004). Decreased absolute amygdala volume in cocaine addicts. Neuron 44: 729-740.

McFarland BR, Shankman SA, Tenke CE, Bruder GE, Klein DN (2006). Behavioral activation system deficits predict the sixmonth course of depression. J Affect Disord 91: 229-234.

Morey RA, Petty CM, Xu Y, Hayes JP, Wagner HR 2nd, Lewis DV et al (2009). A comparison of automated segmentation and manual tracing for quantifying hippocampal and amygdala volumes. NeuroImage 45: 855-866.

Petry NM (2001). Pathological gamblers, with and without substance use disorders, discount delayed rewards at high rates. J Abnorm Psychol 110: 482-487.

Potenza MN (2008). The neurobiology of pathological gambling and drug addiction: an overview and new findings. Philos Trans $R$ Soc Lond B Biol Sci 363: 3181-3189.

Potenza MN, Balodis IM, Franco CA, Bullock S, Xu J, Chung T et al (2013). Neurobiological considerations in understanding behavioral treatments for pathological gambling. Psychol Addict Behav 27: 380-392.

Potenza MN, Kosten TR, Rounsaville BJ (2001). Pathological gambling. JAMA 286: 141-144.

Potenza MN, Steinberg MA, Skudlarski P, Fulbright RK, Lacadie CM, Wilber MK et al (2003). Gambling urges in pathological gambling: a functional magnetic resonance imaging study. Arch Gen Psychiatry 60: 828-836.

Potenza MN, Xian H, Shah K, Scherrer JF, Eisen SA (2005). Shared genetic contributions to pathological gambling and major depression in men. Arch Gen Psychiatry 62: 1015-1021.
Rahman AS, Pilver CE, Desai RA, Steinberg MA, Rugle L, Krishnan-Sarin S et al (2012). The relationship between age of gambling onset and adolescent problematic gambling severity. J Psychiatr Res 46: 675-683.

Rando K, Tuit K, Hannestad J, Guarnaccia J, Sinha R (2013). Sex differences in decreased limbic and cortical grey matter volume in cocaine dependence: a voxel-based morphometric study. Addict Biol 18: 147-160.

Rusch BD, Abercrombie HC, Oakes TR, Schaefer SM, Davidson RJ (2001). Hippocampal morphometry in depressed patients and control subjects: relations to anxiety symptoms. Biol Psychiatry 50: $960-964$.

Shaffer HJ, Korn DA (2002). Gambling and related mental disorders: a public health analysis. Annu Rev Public Health 23: 171-212.

Shen L, Saykin AJ, Kim S, Firpi HA, West JD, Risacher SL et al (2010). Comparison of manual and automated determination of hippocampal volumes in MCI and early AD. Brain Imaging Behav 4: 86-95.

Tanabe J, Thompson L, Claus E, Dalwani M, Hutchison K, Banich MT (2007). Prefrontal cortex activity is reduced in gambling and nongambling substance users during decision-making. Hum Brain Mapp 28: 1276-1286.

van Holst RJ, van den Brink W, Veltman DJ, Goudriaan AE (2010). Why gamblers fail to win: a review of cognitive and neuroimaging findings in pathological gambling. Neurosci Biobehav Rev 34: 87-107.

van Toor D, Roozen HG, Evans BE, Rombout L, Van de Wetering BJ, Vingerhoets AJ (2011). The effects of psychiatric distress, inhibition, and impulsivity on decision making in patients with substance use disorders: A matched control study. J Clin Exp Neuropsychol 33: 161-168.

Yip SW, Lacadie C, Xu J, Worhunsky PD, Fulbright RK, Constable RT et al (2013). Reduced genual corpus callosal white matter integrity in pathological gambling and its relationship to alcohol abuse or dependence. World J Biol Psychiatry 14: 129-138.

Zetzsche T, Preuss UW, Frodl T, Schmitt G, Seifert D, Munchhausen $\mathrm{E}$ et al (2007). Hippocampal volume reduction and history of aggressive behaviour in patients with borderline personality disorder. Psychiatry Res 154: 157-170.

Supplementary Information accompanies the paper on the Neuropsychopharmacology website (http://www.nature.com/npp) 